\title{
POLYPHENOLIC COMPOUNDS FROM THE LEAVES OF SCHINUS TEREBINTHIFOLIUS RADDI
}

Salwa F. Farag

Department of Pharmacognosy, Faculty of Pharmacy, Assiut University, 71526, Assiut, Egypt

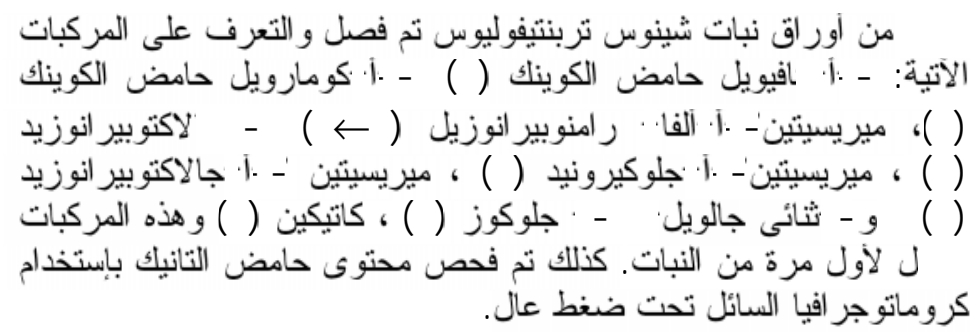

Two quinic acid esters, 5-O-caffeoylquinic acid (1) and 5-Ocoumaroylquinic acid (2); three myricetin glycosides, myricetin 3$O$ - $\alpha$-L-rhamnopyranosyl $\left(1^{\prime \prime \prime} \rightarrow 6^{\prime \prime}\right) \beta$-D-galactopyranoside (3), myricetin 3-O- $\beta$-D-glucuronide (4), and myricetin 3-O- $\beta$-Dgalactopyranoside (5); 1,6-digalloyl- $\beta$-D-glucose (6); and (+)catechin (7) were isolated and identified for the first time from the leaves of Schinus terebinthifolius Raddi. Furthermore, investigation of tannic acid content was carried out by HPLC.

\section{INTRODUCTION}

The family Anacardiaceae comprises about 600 species belonging to 70 genera. The plants of this family are mainly tropical trees and shrubs used for dyeing and tanning. The genus Schinus is a member of this family, which includes 30 species $^{1}$. The tree of Schinus terebinthifolius Raddi (pepper tree) is evergreen, has leathery imparipinnate leaves, with an abudance of small flowers formed in panicles. The fruits are small brightred drupes ${ }^{2}$. The bark of $S$. terebinthifolius Raddi is used for dressing leather and a dye is also extracted from it $^{2}$. The extract of the stem bark is used as an antiinflammatory and wound-healing agent ${ }^{3}$. The leaves and stem bark are used as tonic, to treat wounds, and urinary and respiratory tract 
infections. They have antiseptic, antiinflammatory, balsamic, haemostatic, and antioxidant activities ${ }^{4}$ The essential oil of the plant is used to treat respiratory problems, mycosis, and candidal infections (topical use); its activity is attributed to the presence of high concentrations of monoterpenes in the plant ${ }^{4}$. Ethanolic extracts from the leaves, stem bark, and fruits exhibited antimicrobial activity $^{4-6}$. Pentagalloylglucose isolated from the aerial parts of this plant has been reported to exhibit inhibitory activity against xanthine oxidase ${ }^{7}$. Polyphenols purified from the leaves of the plant induced anti-proliferative effect ${ }^{8}$. Triterpenoids present in the berries have been shown to act as specific competitive inhibitors of secreted phospholipase- $\mathrm{A}_{2}{ }^{9}$.

Leaves and bark of the pepper tree contain tannins and essential oil ${ }^{10}$. The results of the chemical analysis of the stem bark showed the presence of catechin, saponins, terpenes, and flavonoids ${ }^{3}$. Phytochemical study of the drupes led to the isolation of apigenin, naringin, ellagic, syringic acid ${ }^{5}$ biflavonoids, gallic acid ${ }^{11}$, penta galloylglucose ${ }^{7}, \quad n$-alkylphenols ${ }^{12}$, and cardanol ${ }^{13}$.

This paper deals with the isolation and identification of seven polyphenolic compounds including, 5-O-caffeoylquinic acid (1), 5-Ocoumaroylquinic acid (2), myricetin 3 - $O$ - $\alpha$-L-rhamnopyranosyl $\left(1^{\prime \prime \prime} \rightarrow 6 "\right) \beta$ D-galactopyranoside (3), myricetin 3$O$ - $\beta$-D-glucuronide (4), myricetin 3$O-\beta$-D-galactopyranoside (5), 1,6digalloyl- $\beta$-D-glucose (6), and (+)- catechin (7) from the leaves of $S$. terebinthifolius Raddi. Moreover, investigation of tannic acid content was carried out by normal-phase HPLC.

\section{EXPERIMENTAL}

\section{General procedures}

NMR experiments were recorded in $\mathrm{CD}_{3} \mathrm{OD}$ and DMSO- $d_{6}$ using a Varian Unity Inova AS600NB spectrometer $\left(600 \mathrm{MHz}\right.$ for ${ }^{1} \mathrm{H}$ NMR and $150 \mathrm{MHz}$ for ${ }^{13} \mathrm{C}$ NMR). UV spectra were measured with a JASCO V-530 UV-VIS spectrophotometer (Jasco, Tokyo, Japan). The IR (KBr) spectra were taken on a JASCO FT/IR-410 spectrophotometer. Column chromatography was carried out on Toyopearl HW-40 (coarse grade; Tosoh Company, Tokyo, Japan), Diaion HP-20 (Mitsubishi Chemical Industries, Tokyo, Japan) and MCI-gel CHP-20P (75-150 $\mu \mathrm{m}$, Mitsubishi Kasei Company, Tokyo, Japan). Preparative TLC was performed on Kieselgel $60 \mathrm{~F}_{254}$ plates (layer thickness of $0.5 \mathrm{~mm}$; Merck, Darmstadt, Germany), while analytical TLC was conducted on precoated aluminium sheets of silica gel $60 \mathrm{GF}_{254}$ (Merck, Darmstadt, Germany) using $\mathrm{CHCl}_{3}-\mathrm{MeOH}-\mathrm{H}_{2} \mathrm{O}$ (65:30:5). The spots were detected by UV irradiation (254 and $366 \mathrm{~nm}$ ) followed by spraying with $10 \%$ $\mathrm{H}_{2} \mathrm{SO}_{4}$ reagent or $5 \% \quad \mathrm{AlCl}_{3}$. Authentic samples of chlorogenic acid, tannic acid and 1,2,3,4,6pentagalloyl- $\beta$-D-glucose were obtained from Division of 
Pharmaceutical Sciences, Okayama University Graduate School of Medicine, Dentistry and Pharmaceutical Sciences, Tsushima, Okayama 700-8530, Japan.

\section{Plant material}

The leaves of Schinus terebinthifolius Raddi were collected during the flowering stage in July 2006 from the Experimental Station of Medicinal Plants, Faculty of Agriculture, Assiut University, Assiut, Egypt. The plant was identified by Prof. Dr. Naeem ElKeltawy, Prof. of Horticulture, Faculty of Agriculture, Assiut University. A voucher sample has been deposited in the Herbarium of Pharmacognosy Department, Faculty of Pharmacy, Assiut University, Assiut, Egypt.

\section{Extraction and isolation}

Air-dried powdered leaves of $S$. terebinthifolius Raddi $(2.0 \mathrm{~kg}$ ) were extracted with $70 \%$ aqueous acetone $(3 \times 6 \mathrm{~L})$ and the combined extracts filtered and concentrated. The extract was suspended in $\mathrm{H}_{2} \mathrm{O}\left(\begin{array}{ll}1 & \mathrm{~L}\end{array}\right)$ and successively partitioned with $\mathrm{Et}_{2} \mathrm{O}$ $(3 \times 1 \mathrm{~L})$, EtOAc $(3 \times 1 \mathrm{~L})$, and $n$ $\mathrm{BuOH}$ saturated with $\mathrm{H}_{2} \mathrm{O}(3 \times 1 \mathrm{~L})$. Each phase was concentrated under reduced pressure to give the corresponding soluble fraction (17.1 g), $(114.7 \mathrm{~g})$ and $(73.0 \mathrm{~g})$, respectively, in addition to $14.5 \mathrm{~g}$ as water soluble residue.

About $63.0 \mathrm{~g}$ of the $n-\mathrm{BuOH}$ fraction was applied to a column of Diaion HP-20 and eluted successively with $\mathrm{H}_{2} \mathrm{O}, 20 \% \mathrm{MeOH}, 40 \% \mathrm{MeOH}$, $60 \% \mathrm{MeOH}$ and $100 \% \mathrm{MeOH}$ to yield 5 fractions, B-I (24.0 g), B-II (4.5 g), B-III (10.4 g), B-IV (17.9 g), and B-V (4.0 g), respectively. About $2.3 \mathrm{~g}$ of fraction B-II was chromatographed over Toyopearl HW-40 (coarse grade) column $(40 \times$ $2.2 \mathrm{~cm}$ ) and eluted with $\mathrm{MeOH}-\mathrm{H}_{2} \mathrm{O}$ gradient to yield 5 fractions: B-II-1 $(2: 8,300.0 \mathrm{mg})$, B-II-2 (3:7, 137.9 $\mathrm{mg})$, B-II-3 (4:6, $36.8 \mathrm{mg})$, B-II-4 (5:5, $102.7 \mathrm{mg})$, and B-II-5 (MeOH, $1.6 \mathrm{~g})$. Fraction B-II-2 was purified on MCI-gel CHP-20P column $(48 \times$ $1.1 \mathrm{~cm}$ ) using $\mathrm{MeOH}-\mathrm{H}_{2} \mathrm{O}$ (1:4) and $\mathrm{MeOH}-\mathrm{H}_{2} \mathrm{O}$ (3:7) as eluents to afford compounds 1 (37.0 $\mathrm{mg}$ ) and 2 (27.0 $\mathrm{mg}$ ), respectively. Fraction B-II-3 was subjected to preparative TLC to yield compounds $3(8.4 \mathrm{mg})$ and $7(9.5$ $\mathrm{mg}$ ), respectively. Fraction B-II-4 was purified on MCI-gel CHP-20P column and eluted with $\mathrm{MeOH}-\mathrm{H}_{2} \mathrm{O}$ (3:7) to yield compound 6 ( $3 \mathrm{mg}$ ) followed by $\mathrm{MeOH}-\mathrm{H}_{2} \mathrm{O}$ (2:3) to yield compounds $4(18.3 \mathrm{mg})$ and $\mathbf{5}$ (8.0 mg).

Compound 1: Yellowish brown amorphous powder; $\mathrm{R}_{\mathrm{f}}=0.17$; IR $v_{\max }$ (KBr): 3375, 1706, 1677, 1627, 1595, $1508,1433 \mathrm{~cm}^{-1} ;{ }^{1} \mathrm{H}$ NMR $\left(\mathrm{CD}_{3} \mathrm{OD}\right.$, $600 \mathrm{MHz}): \delta 2.10 \sim 2.25\left(4 \mathrm{H}, \mathrm{m}, \mathrm{H}_{2-}\right.$ 2/6), $3.77(1 \mathrm{H}, \mathrm{dd}, \mathrm{J}=9.0,3.6 \mathrm{~Hz}, \mathrm{H}-$ 4), $4.21(1 \mathrm{H}, \mathrm{m}, \mathrm{H}-3), 5.38(1 \mathrm{H}, \mathrm{ddd}$, $\mathrm{J}=13.8,9.0,4.2 \mathrm{~Hz}, \mathrm{H}-5), 6.30(1 \mathrm{H}$, $\left.\mathrm{d}, \mathrm{J}=16.2 \mathrm{~Hz}, \mathrm{H}-2^{\prime}\right), 6.82(1 \mathrm{H}, \mathrm{d}, \mathrm{J}=$ $\left.8.4 \mathrm{~Hz}, \mathrm{H}-8^{\prime}\right), 6.99(1 \mathrm{H}, \mathrm{dd}, \mathrm{J}=8.4$, $\left.2.4 \mathrm{~Hz}, \mathrm{H}-9^{\prime}\right), 7.09(1 \mathrm{H}, \mathrm{d}, \mathrm{J}=2.4 \mathrm{~Hz}$, $\left.\mathrm{H}-5^{\prime}\right), 7.60\left(1 \mathrm{H}, \mathrm{d}, \mathrm{J}=16.2 \mathrm{~Hz}, \mathrm{H}-3^{\prime}\right)$; ${ }^{13} \mathrm{C}$ NMR $\left(\mathrm{CD}_{3} \mathrm{OD}, 150 \mathrm{MHz}\right)$ : 
<smiles>[R6]C1C[C@](O)(C(=O)O)C[C@@H](O)[C@@H]1O</smiles>

$1: \mathrm{R}=$ Caffeoyl

$2: \mathrm{R}=p$-Coumaroyl<smiles></smiles>

$3: R=$ Gal $\left(6^{\prime \prime-1}{ }^{\prime \prime \prime}\right)$ Rha

4: $\mathrm{R}=\mathrm{GlcA}$

$5: \mathrm{R}=\mathrm{Gal}$

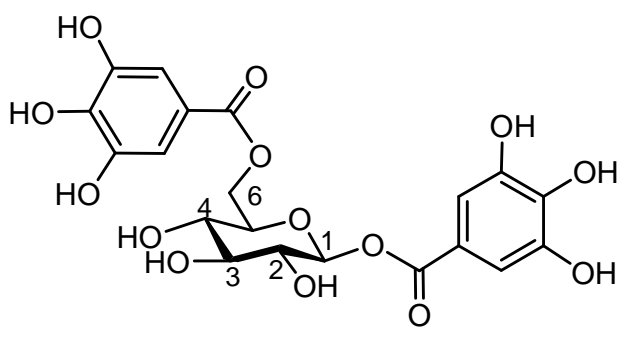

6<smiles>CC(=O)C=CC=COC(C)=O</smiles><smiles>OC[C@H]1O[C@H](CO)[C@H](O)[C@@H](O)[C@@H]1O</smiles>

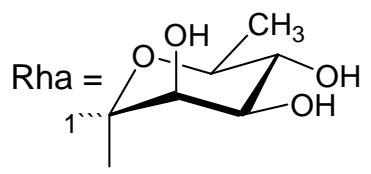

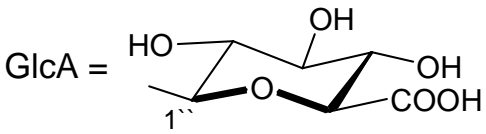<smiles>Oc1cc(O)c2c(c1)O[C@H](c1ccc(O)c(O)c1)[C@H](O)C2</smiles>

7

Structures of compounds 1-7.

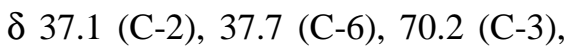
70.8 (C-5), 72.4 (C-4), 75.0 (C-1), $114.1\left(\mathrm{C}-2^{\prime} / 5^{\prime}\right), 115.3$ (C-8'), 121.8 $\left(\mathrm{C}-9^{\prime}\right), 126.6\left(\mathrm{C}-4^{\prime}\right), 145.6\left(\mathrm{C}-6^{\prime}\right)$, 145.9 (C-3'), 148.4 (C-7'), 167.5 (C1'), 175.9 (C-7).
Compound 2: Yellowish brown amorphous powder; $\mathrm{R}_{\mathrm{f}}=0.28$; IR $v_{\max }$ (KBr): 3400, 1706, 1682, 1631, 1596, 1504, $1433 \mathrm{~cm}^{-1}$; ${ }^{1} \mathrm{H}$ NMR (DMSO$\left.d_{6}, 600 \mathrm{MHz}\right): \delta 1.79 \sim 1.98(4 \mathrm{H}, \mathrm{m}$, $\left.\mathrm{H}_{2}-2 / 6\right), 3.56(1 \mathrm{H}, \mathrm{m}, \mathrm{H}-4), 3.93(1 \mathrm{H}$, 
m, H-3), $5.08(1 \mathrm{H}, \mathrm{m}, \mathrm{H}-5), 6.27(1 \mathrm{H}$, d, J= $\left.16.2 \mathrm{~Hz}, \mathrm{H}-2^{\prime}\right), 6.79(2 \mathrm{H}, \mathrm{d}, \mathrm{J}=$ $\left.8.4 \mathrm{~Hz}, \mathrm{H}-6^{\prime} / 8^{\prime}\right), 7.49(1 \mathrm{H}, \mathrm{d}, \mathrm{J}=16.2$ $\left.\mathrm{Hz}, \mathrm{H}-3^{\prime}\right), 7.52(2 \mathrm{H}, \mathrm{d}, \mathrm{J}=8.4 \mathrm{~Hz}, \mathrm{H}-$ $\left.5^{\prime} / 9^{\prime}\right) ;{ }^{13} \mathrm{C}$ NMR (DMSO- $d_{6}, 150$ MHz): $\delta 41.8$ (C-2), 42.6 (C-6), 73.6 (C-4), 75.9 (C-3), 76.3 (C-5), 78.9 (C-1), 119.9 (C-2'), $121.2\left(\mathrm{C}-6^{\prime} / 8^{\prime}\right)$, 130.6 (C-4'), 135.7 (C-5'/9'), 149.9 $\left(\mathrm{C}-3^{\prime}\right), 165.2\left(\mathrm{C}-7^{\prime}\right), 171.2\left(\mathrm{C}-1^{\prime}\right)$, $180.3(\mathrm{C}-7)$.

Compound 3: Yellowish brown amorphous powder; $\mathrm{R}_{\mathrm{f}}=0.32$; $\mathrm{UV}$ $(\mathrm{MeOH}) \lambda_{\max }: 258,301 \mathrm{sh}, 363$; + NaOMe: 268, 322, 408; + $\mathrm{AlCl}_{3}$ : 270, 310 sh, $437 ;+\mathrm{AlCl}_{3} / \mathrm{HCl}: 272,307$, 415; + NaOAc: 270, 321, 408; + $\mathrm{NaOAc} / \mathrm{H}_{3} \mathrm{BO}_{3}$ : $258,386 \mathrm{~nm} .{ }^{1} \mathrm{H}$ NMR $\left(\mathrm{CD}_{3} \mathrm{OD}, 600 \mathrm{MHz}\right): \delta 1.27$ $\left(3 \mathrm{H}, \mathrm{d}, \mathrm{J}=6.6 \mathrm{~Hz}, \mathrm{H}-6^{\prime \prime \prime}\right), 3.48(1 \mathrm{H}$, dd, J=10.2, 7.2 Hz, H-6"b), $3.80(1 \mathrm{H}$, dd, J=10.2, 6.0 Hz, H-6"a), 3.54 3.90 (m, other sugar protons), $4.58(1 \mathrm{H}, \mathrm{d}$, $\left.\mathrm{J}=1.8 \mathrm{~Hz}, \mathrm{H}-1^{\prime \prime \prime}\right), 5.10(1 \mathrm{H}, \mathrm{d}, \mathrm{J}=7.8$ $\left.\mathrm{Hz}, \mathrm{H}-1^{\prime \prime}\right), 6.21(1 \mathrm{H}, \mathrm{d}, \mathrm{J}=1.8 \mathrm{~Hz}, \mathrm{H}-$ 6), $6.40(1 \mathrm{H}, \mathrm{d}, \mathrm{J}=1.8 \mathrm{~Hz}, \mathrm{H}-8)$, $7.45\left(2 \mathrm{H}, \mathrm{s}, \mathrm{H}-2^{\prime} / \mathrm{H}-6^{\prime}\right) ;{ }^{13} \mathrm{C} \mathrm{NMR}$ ( $\left.\mathrm{CD}_{3} \mathrm{OD}, 150 \mathrm{MHz}\right): \delta 18.0$ (C-6"'), 67.4 (C-6"), 69.7 (C-5"'), 70.2 (C-4"), 72.1 (C-3"), 72.3 (C-3'"), 73.2 (C-2"), 73.9 (C-4"'), 75.2 (C-2'"), 75.3 (C5"), 95.4 (C-8), 100.8 (C-6), 102.0 (C-1'"), 104.8 (C-10), 106.4 (C-1"), $110.1\left(\mathrm{C}-2^{\prime} / 6^{\prime}\right), 121.6\left(\mathrm{C}-1^{\prime}\right), 136.0$ (C-3), 138.3 (C-4'), 146.4 (C-3'/5'), 158.5 (C-2), 158.6 (C-9), 162.8 (C-5), 168.7 (C-7), 179.0 (C-4).

Compound 4: Yellow amorphous powder; $\mathrm{R}_{\mathrm{f}}=0.14$; $\mathrm{UV}(\mathrm{MeOH}) \lambda_{\max }$ :
259, 302 sh, 365 ; + NaOMe: 269, 324, 410; + $\mathrm{AlCl}_{3}:$ 270, 308 sh, 430; $+\mathrm{AlCl}_{3} / \mathrm{HCl}:$ 272, 307, 408; + NaOAc: 270, 326, 400; + NaOAc/ $\mathrm{H}_{3} \mathrm{BO}_{3}: 259, \quad 380 \mathrm{~nm} .{ }^{1} \mathrm{H} \quad \mathrm{NMR}$ (DMSO- $d_{6}, 600 \mathrm{MHz}$ ): $\delta$ 3.24 3.42 $(\mathrm{m}$, sugar protons $), 3.54(1 \mathrm{H}, \mathrm{d}, \mathrm{J}=$ $\left.10.2 \mathrm{~Hz}, \mathrm{H}-5^{\prime \prime}\right), 5.49(1 \mathrm{H}, \mathrm{d}, \mathrm{J}=7.8$ $\left.\mathrm{Hz}, \mathrm{H}-1^{\prime \prime}\right), 6.19(1 \mathrm{H}, \mathrm{d}, \mathrm{J}=1.8 \mathrm{~Hz}, \mathrm{H}-$ 6), $6.37(1 \mathrm{H}, \mathrm{d}, \mathrm{J}=1.8 \mathrm{~Hz}, \mathrm{H}-8), 7.19$ $\left(2 \mathrm{H}, \mathrm{s}, \mathrm{H}-2^{\prime} / 6^{\prime}\right) ;{ }^{13} \mathrm{C}$ NMR (DMSO$\left.d_{6}, 150 \mathrm{MHz}\right): \delta 71.4$ (C-4"), 73.8 (C$\left.2^{\prime \prime}\right), 76.1$ (C-5"), 76.2 (C-3"), 93.6 (C8), 98.9 (C-6), 101.3 (C-10), 104.0 (C-1"), 108.7 (C-2'/6'), 119.9 (C-1'), 133.5 (C-3), $137.0\left(\mathrm{C}-4^{\prime}\right), 145.6$ (C3'/5'), 156.3 (C-9), 156.4 (C-2), 161.4 (C-5), 164.4 (C-7), 170.0 (C-6"), $177.3(\mathrm{C}-4)$.

Compound 5: Yellow amorphous powder; $\mathrm{R}_{\mathrm{f}}=0.41$; $\mathrm{UV}(\mathrm{MeOH}) \lambda_{\max }$ : 260, 300 sh, 363; + NaOMe: 270, 322, 408; $+\mathrm{AlCl}_{3}: 272,308$ sh, 437; $+\mathrm{AlCl}_{3} / \mathrm{HCl}: 274,307,415$; + NaOAc: 272, 322, 408; + NaOAcl $\mathrm{H}_{3} \mathrm{BO}_{3}: \quad 260,386 \mathrm{~nm} .{ }^{1} \mathrm{H} \quad \mathrm{NMR}$ (DMSO- $d_{6}, 600 \mathrm{MHz}$ ): $\delta$ 3.27 3.63 (m, sugar protons), $5.30(1 \mathrm{H}, \mathrm{d}, \mathrm{J}=$ $\left.7.8 \mathrm{~Hz}, \mathrm{H}-1^{\prime \prime}\right), 6.11(1 \mathrm{H}, \mathrm{d}, \mathrm{J}=1.8 \mathrm{~Hz}$, H-6), $6.29(1 \mathrm{H}, \mathrm{d}, \mathrm{J}=1.8 \mathrm{~Hz}, \mathrm{H}-8)$, $7.19\left(2 \mathrm{H}, \mathrm{s}, \mathrm{H}-2^{\prime} / \mathrm{H}-6^{\prime}\right) ;{ }^{13} \mathrm{C}$ NMR (DMSO- $d_{6}, 150 \mathrm{MHz}$ ): $\delta 60.2$ (C-6"), 68.1 (C-4"), 71.4 (C-2"), 73.5 (C-3"), 76.1 (C-5"), 93.7 (C-8), 99.2 (C-6), 102.4 (C-1"), 103.5 (C-10), 108.7 (C$\left.2^{\prime} / 6^{\prime}\right), 120.0 \quad\left(\mathrm{C}-1^{\prime}\right), 133.8 \quad(\mathrm{C}-3)$, 137.1 (C-4'), 145.6 (C-3'/5'), 156.1 (C-2), 156.5 (C-9), 161.3 (C-5), 165.6 (C-7), 177.3 (C-4). 
Compound 6: White amorphous powder; $\mathrm{R}_{\mathrm{f}}=0.3 ;{ }^{1} \mathrm{H}$ NMR $\left(\mathrm{CD}_{3} \mathrm{OD}\right.$, $600 \mathrm{MHz}): \delta 3.55 \sim 3.75(4 \mathrm{H}, \mathrm{m}, \mathrm{H}-$ $\left.2^{\prime}, 3^{\prime}, 4^{\prime}, 5^{\prime}\right), 4.44(1 \mathrm{H}, \mathrm{dd}, \mathrm{J}=12.0,5.4$ $\left.\mathrm{Hz}, \mathrm{H}-6^{\prime} \mathrm{b}\right), 4.59$ (1H, dd, J=12.0, 1.8 Hz, H-6'a), 5.73 (1H, d, J= $7.8 \mathrm{~Hz}, \mathrm{H}-$ 1'), 7.12, 7.17 (each 2H, s, galloyl-H); ${ }^{13} \mathrm{C}$ NMR $\left(\mathrm{CD}_{3} \mathrm{OD}, 150 \mathrm{MHz}\right): \delta$ 63.3 (C-6'), 70.0 (C-4'), 72.9 (C-2'), 75.3 (C-3'), 76.9 (C-5'), 94.8 (C-1'), 109.0, 109.4 (galloyl C-2/6), 119.9, 120.2 (galloyl C-1), 139.2, 139.5 (galloyl C-4), 145.3 (galloyl C-3/5), 165.8, 167.1 (galloyl-CO).

Compound 7: White amorphous powder; $\mathrm{R}_{\mathrm{f}}=0.74 ;{ }^{1} \mathrm{H} \mathrm{NMR}\left(\mathrm{CD}_{3} \mathrm{OD}\right.$, $600 \mathrm{MHz}): \delta 2.55(1 \mathrm{H}, \mathrm{dd}, \mathrm{J}=16.2$, $7.8 \mathrm{~Hz}, \mathrm{H}-4 \mathrm{~b}), 2.89$ (1H, dd, J= 16.2, $5.4 \mathrm{~Hz}, \mathrm{H}-4 \mathrm{a}), 4.02(1 \mathrm{H}$, ddd, J= 7.8, 7.2, $5.4 \mathrm{~Hz}, \mathrm{H}-3), 4.60(1 \mathrm{H}, \mathrm{d}, \mathrm{J}=7.2$ $\mathrm{Hz}, \mathrm{H}-2), 5.90(1 \mathrm{H}, \mathrm{d}, \mathrm{J}=1.8 \mathrm{~Hz}, \mathrm{H}-$ 6), $5.97(1 \mathrm{H}, \mathrm{d}, \mathrm{J}=1.8 \mathrm{~Hz}, \mathrm{H}-8), 6.75$ $\left(1 \mathrm{H}, \mathrm{dd}, \mathrm{J}=8.4,2.4 \mathrm{~Hz}, \mathrm{H}-6^{\prime}\right), 6.80$ $\left(1 \mathrm{H}, \mathrm{d}, \mathrm{J}=8.4 \mathrm{~Hz}, \mathrm{H}-5^{\prime}\right), 6.88(1 \mathrm{H}, \mathrm{d}$, $\mathrm{J}=2.4 \mathrm{~Hz}, \mathrm{H}-2^{\prime}$ ).

\section{Determination of the tannic acid content \\ Chromatographic equipment and conditions}

Normal-phase HPLC was conducted on a YMC-Pack SIL A003 column $(250 \mathrm{~mm} \times 4.6 \mathrm{~mm}$ i.d.; YMC, Kyoto, Japan) developed with $n$-hexane-MeOH-THF-formic acid 60:45:15:1 containing oxalic acid 500 $\mathrm{mg} / 1.2 \mathrm{~L}$ (flow rate, $1.5 \mathrm{~m} \mathrm{~L} / \mathrm{min}$; UV detection, $280 \mathrm{~nm}$ ) at room temperature.

\section{Sample preparation}

One milligram each of EtOAc and $n$ - $\mathrm{BuOH}$-soluble fraction as well as a reference sample of pentagalloylglucose $\left(\mathrm{R}_{\mathrm{t}}=6.45 \mathrm{~min}\right)$ was dissolved in $1 \mathrm{~mL} \mathrm{MeOH}$. Aliquots $(2 \mathrm{~L})$ of each solution were subjected to HPLC and the areas under the peaks were recorded.

\section{RESULTS AND DISCUSSION}

Seventy percent aqueous acetone extract obtained from the leaves of $S$. terebinthifolius Raddi was suspended in $\mathrm{H}_{2} \mathrm{O}$ and partitioned with $\mathrm{Et}_{2} \mathrm{O}$, EtOAc, and $n$-BuOH. The $n-\mathrm{BuOH}$ soluble fraction was subjected to Diaion HP-20 column chromatography using $\mathrm{H}_{2} \mathrm{O}, 20 \% \mathrm{MeOH}, 40 \%$ $\mathrm{MeOH}, 60 \% \mathrm{H}_{2} \mathrm{O}$, and $\mathrm{MeOH}$ as eluates, successively. Normal-phase HPLC analysis revealed that the fraction eluted with $20 \% \mathrm{MeOH}$ contained the least amount of tannic acid when compared to the EtOAc and other $n-\mathrm{BuOH}$ fractions. This fraction was separated by repeatedcolumn chromatography on Toyopearl HW-40 (coarse grade) and MCI-gel CHP-20P and preparative TLC to yield seven phenolic compounds (1-7).

Compound 1 was obtained as yellowish brown amorphous powder. Its ${ }^{1} \mathrm{H}$ NMR spectrum showed two doublets at $\delta 6.30$ and 7.60 with coupling constants of $16.2 \mathrm{~Hz}$ due to trans olefinic protons in addition to three doublets at $\delta 6.82(1 \mathrm{H}, \mathrm{d}, \mathrm{J}=8.4$ $\mathrm{Hz}), 6.99(1 \mathrm{H}, \mathrm{dd}, \mathrm{J}=8.4,2.4 \mathrm{~Hz})$ and $7.09(1 \mathrm{H}, \mathrm{d}, \mathrm{J}=2.4 \mathrm{~Hz})$ assigned to 
one 1,3,4-trisubstituted aromatic ring moiety. Also, the ${ }^{13} \mathrm{C}$ NMR spectrum showed signals at $\delta 114.1,115.3$, $121.8,126.6,145.6,145.9,148.4$, and 167.5. These observations suggested the presence of a caffeic acid unit ${ }^{14}$. Moreover, signals of three oxymethine protons appeared at $\delta_{\mathrm{H}}$ $3.77(1 \mathrm{H}, \mathrm{dd}, \mathrm{J}=9.0,3.6 \mathrm{~Hz}), 4.21$ $(1 \mathrm{H}, \mathrm{m})$, and $5.38(1 \mathrm{H}, \mathrm{ddd}, \mathrm{J}=13.8$, $9.0,4.2 \mathrm{~Hz}$ ) in addition to a broad signal for two methylene groups at $\delta$ 2.10 2.25 $(4 \mathrm{H}, \mathrm{m})$ suggesting the presence of one quinic acid moiety ${ }^{14}$. This assignment was supported by the analysis of ${ }^{13} \mathrm{C}$ NMR spectral data that displayed three oxymethines $(\delta$ $70.2,70.8$, and 72.4), two methylenes ( $\delta 37.1$ and 37.7 ), one oxygenated quaternary carbon $(\delta 75.0)$, and one carboxyl carbon $(\delta$ 175.9). The location of caffeoyl substitution on the quinic acid moiety was deduced from the downfield shift of the proton at $\mathrm{C}-5(\delta$ 5.38) compared to free quinic acid ${ }^{15}$. By direct comparison with literature data ${ }^{16}$ and authentic sample (co-TLC), the structure of compound 1 was identified as 5-Ocaffeoylquinic acid (chlorogenic acid) $(0.0019 \%)$.

Compound 2 was isolated as yellowish brown amorphous powder. Its ${ }^{1} \mathrm{H}$ and ${ }^{13} \mathrm{C}$ NMR spectral data are very close to those of $\mathbf{1}$ except for the appearance of one $p$-coumaric acid unit instead of caffeic acid unit. This was indicated by the appearance of two characteristic doublets for trans olefinic protons at $\delta_{\mathrm{H}} 6.27$ and 7.49 (each $2 \mathrm{H}, \mathrm{J}=16.2 \mathrm{~Hz}$ ), in addition to two doublets for 1,4-disubstituted aromatic ring at $\delta_{\mathrm{H}} 6.79$ and 7.52 (each $2 \mathrm{H}, \mathrm{J}=8.4 \mathrm{~Hz})^{14}$. Furthermore, the ${ }^{13} \mathrm{CNMR}$ spectrum displayed characteristic signals at $\delta$ 119.9, $121.2,130.6,135.7,149.9,165.2$, and 171.2. Thus, the structure of compound 2 was identified as 5-Ocoumaroylquinic acid $(0.0014 \%)$ by comparison of its spectral data with those published in the literature ${ }^{17}$.

The UV spectral data in methanol for compounds 3-5 suggested their structures as $\mathrm{C}-3$ OH substituted flavonols having free hydroxyl groups at positions $5,7,3^{\prime}$ and $4^{\prime 18}$. The ${ }^{1} \mathrm{H}$ NMR spectrum of compound $\mathbf{3}$ revealed a singlet at $\delta 7.45(2 \mathrm{H}, \mathrm{s}, \mathrm{H}-$ $\left.2^{\prime} / 6^{\prime}\right)$ and one set of meta-coupled aromatic protons at $\delta 6.21$ and 6.40 (each $1 \mathrm{H}, \mathrm{d}, \mathrm{J}=1.8 \mathrm{~Hz}$ ) suggesting a myricetin derivative ${ }^{18}$. Furthermore, the appearance of two anomeric protons at $\delta 5.10(1 \mathrm{H}, \mathrm{d}, \mathrm{J}=7.8 \mathrm{~Hz})$ and $4.58(1 \mathrm{H}, \mathrm{d}, \mathrm{J}=1.8 \mathrm{~Hz})$ in addition to a methyl group at $\delta 1.27$ $(3 \mathrm{H}, \mathrm{d}, \mathrm{J}=6.6 \mathrm{~Hz})$ indicated the presence of a $\beta$-sugar unit linked to $\alpha$-rhamnopyranosyl unit $^{19}$. On the basis of the ${ }^{13} \mathrm{C}$ NMR spectral data, the two sugar units were identified as $\beta$-galactopyranosyl and $\alpha$-rhamnopyranosyl units ${ }^{19}$. The downfield shift of C-6" ( $\delta$ 67.4) and the appearance of a cross peak between H-1"' $(\delta$ $4.58)$ and $C-6$ " $(\delta$ 67.4) indicated the interglycosidic linkage $(1 " ' \rightarrow 6 ")$. Also, the HMBC spectrum showed cross peaks between $\mathrm{H}-1$ " $(\delta$ 5.10) and $\mathrm{C}-3(\delta$ 136.0) confirming the glycosylation at postion-3. Based on these data and comparison with 
literature data ${ }^{20}$, compound $\mathbf{3}$ was determined to be myricetin 3-O- $\alpha-\mathrm{L}-$ rhamnopyranosyl $(1 " ' \rightarrow 6$ ") $\beta$-D-galactopyranoside (myricetin 3-robinoside) $(0.0004 \%)$.

The ${ }^{1} \mathrm{H}$ NMR and ${ }^{13} \mathrm{C}$ NMR spectra of compounds $\mathbf{4}$ and $\mathbf{5}$ exhibited signals for myricetin derivative resembling those of $\mathbf{3}$ except for the appearance of $\beta$ glucuronic acid unit $\left(\delta_{\mathrm{C}} 71.4,73.8\right.$, $76.1,76.2,104.0$ and 170.0$)$ and $\beta$ galactopyranosyl unit $\left(\delta_{\mathrm{C}} 60.2,68.1\right.$, $71.4, \quad 73.5, \quad 76.1$ and 102.4), respectively, instead of robinoside unit $^{19}$. By comparison of their spectral data with the reported data ${ }^{21 \& 22}$, the structures of compounds $\mathbf{4}$ and $\mathbf{5}$ were concluded to be myricetin $3-O-\beta-\mathrm{D}$ glucuronide $(0.0009 \%)$ and myricetin 3-O- $\beta$-D-galactopyranoside $(0.0004 \%)$ respectively.

Compound 6 was obtained as white amorphous powder. Its ${ }^{1} \mathrm{H}$ and ${ }^{13} \mathrm{C}$ NMR spectra displayed signals for two galloyl units $\left[\delta_{\mathrm{H}} 7.12\right.$ and 7.17 (each 2H, s); $\delta_{\mathrm{C}} 109.0,109.4$ (C2/6), 119.9, 120.2 (C-1), 139.2, 139.5 (C-4), 145.3 (C-3/5), 165.8 and 167.1 $(\mathrm{CO})]$ and one glucose moiety $\left[\delta_{\mathrm{H}}\right.$ 3.55 3.75 (4H, m, H-2', $\left.3^{\prime}, 4^{\prime}, 5^{\prime}\right), 4.44$ (1H, dd, J= 12.0, $\left.5.4 \mathrm{~Hz}, \mathrm{H}-6^{\prime} \mathrm{b}\right), 4.59$ $\left(1 \mathrm{H}, \mathrm{dd}, \mathrm{J}=12.0,1.8 \mathrm{~Hz}, \mathrm{H}-6^{\prime} \mathrm{a}\right)$ and $5.73\left(1 \mathrm{H}, \mathrm{d}, \mathrm{J}=7.8 \mathrm{~Hz}, \mathrm{H}-1^{\prime}\right) ; \delta_{\mathrm{C}} 63.3$ (C-6'), 70.0 (C-4'), 72.9 (C-2'), 75.3 $\left(\mathrm{C}-3^{\prime}\right), 76.9\left(\mathrm{C}-5^{\prime}\right)$ and $\left.94.8\left(\mathrm{C}-1^{\prime}\right)\right]^{23}$. The large coupling constant $\mathrm{J}=7.8 \mathrm{~Hz}$ indicated the $\beta$-configuration of the anomeric center ${ }^{19}$. The placements of the two galloyl units at C-1 and C-6 were deduced from the downfield shifts of $\mathrm{H}-1^{\prime}(\delta 5.73)$ and $\mathrm{H}-6^{\prime} \mathrm{a}, \mathrm{b}(\delta$ 4.44 and 4.59). From the previous evidence and by comparison of its spectral data with the literature data ${ }^{23}$, compound 6 was assigned as 1,6digalloyl- $\beta$-D-glucose $(0.0002 \%)$.

Compound 7 was isolated as white amorphous powder. The ${ }^{1} \mathrm{H}$ NMR spectrum suggested its nature to be a flavan derivative by the appearance of four signals for the aliphatic protons of ring $\mathrm{C}$ at $\delta 2.55(1 \mathrm{H}, \mathrm{dd}, \mathrm{J}=16.2$, $7.8 \mathrm{~Hz}, \mathrm{H}-4 \mathrm{~b}), 2.89$ (1H, dd, J=16.2, $5.4 \mathrm{~Hz}, \mathrm{H}-4 \mathrm{a}), 4.02(1 \mathrm{H}, \mathrm{ddd}, \mathrm{J}=7.8$, $7.2,5.4 \mathrm{~Hz}, \mathrm{H}-3), 4.60(1 \mathrm{H}, \mathrm{d}, \mathrm{J}=7.2$ $\mathrm{Hz}, \mathrm{H}-2)$. The coupling constant of $\mathrm{H}-2 / \mathrm{H}-3 \quad(\mathrm{~J}=7.8 \mathrm{~Hz})$ indicated its structure as a flavan-3-ol ${ }^{24}$. Furthermore, the spectrum revealed one set of meta-coupled aromatic protons at $\delta 5.90(1 \mathrm{H}, \mathrm{d}, \mathrm{J}=1.8 \mathrm{~Hz}$, H-6), $5.97(1 \mathrm{H}, \mathrm{d}, \mathrm{J}=1.8 \mathrm{~Hz}, \mathrm{H}-8)$ and three aromatic protons of ring $\mathrm{B}$ with a characteristic ABX-type coupling at $\delta 6.75(1 \mathrm{H}, \mathrm{dd}, \mathrm{J}=8.4,2.4$ $\left.\mathrm{Hz}, \mathrm{H}-6^{\prime}\right), 6.80(1 \mathrm{H}, \mathrm{d}, \mathrm{J}=8.4 \mathrm{~Hz}, \mathrm{H}-$ $\left.5^{\prime}\right), 6.88\left(1 \mathrm{H}, \mathrm{d}, \mathrm{J}=2.4 \mathrm{~Hz}, \mathrm{H}-2^{\prime}\right)$. The aforementioned spectral data were in accordance with the data published for (+)-catechin ${ }^{25}$. Thus, compound 7 was identified as (+)-catechin $(0.0004 \%)$.

This is the first report on the occurrence of myricetin glycosides (3-5) in the genus Schinus. Compounds 1, 2 and $\mathbf{6}$ are isolated for the first time from $S$. terebinthifolius Raddi.

The tannic acid content of the EtOAc- and $n$-BuOH-soluble fractions (Fig. 1) from the leaves of 

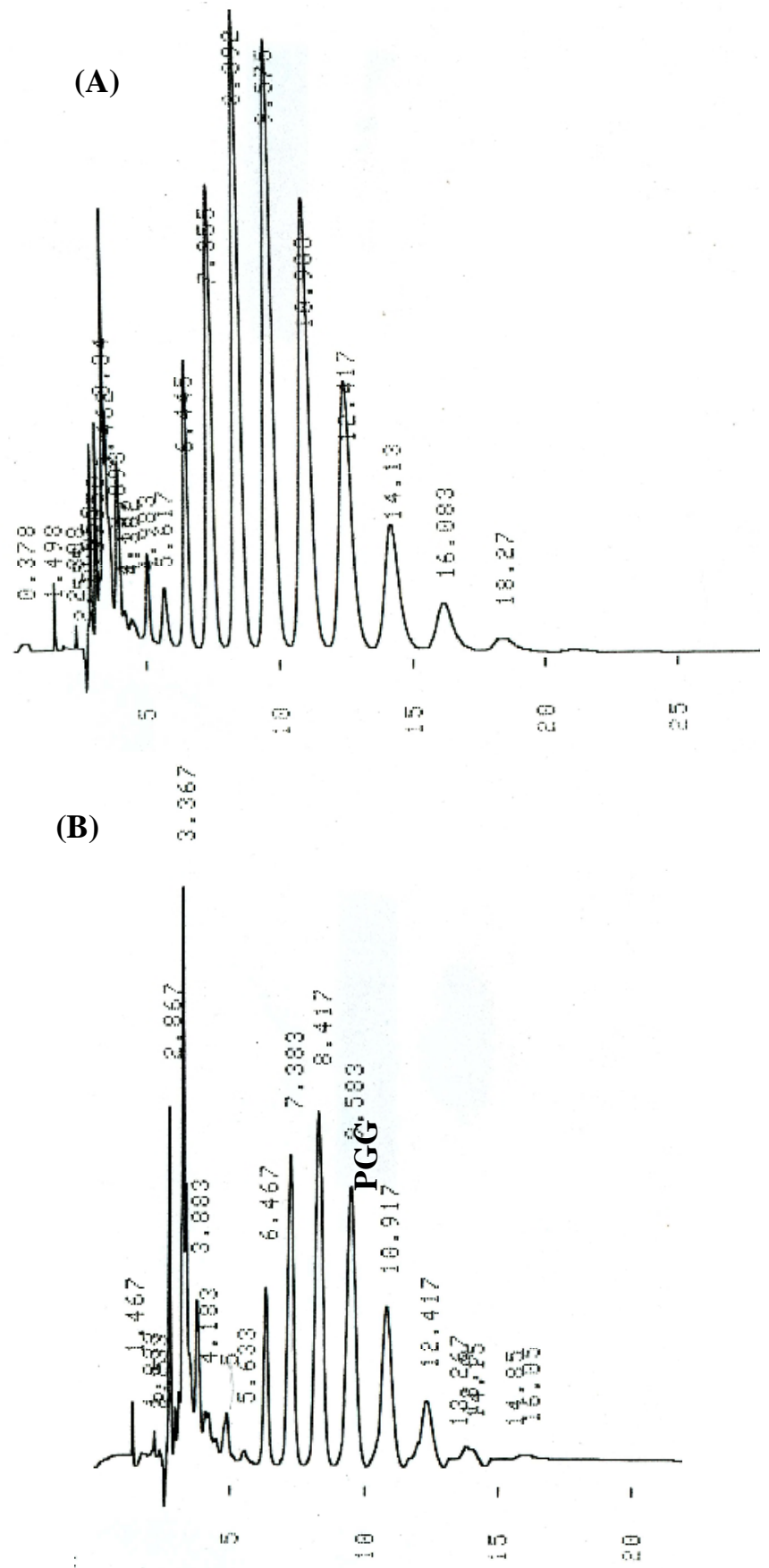

Fig. 1: Normal-phase HPLC investigation of tannic acid content in the leaves of $S$. terebinthifolius Raddi. (A) EtOAc-soluble fraction (B) $n$-BuOH-soluble fraction. 
S. terebinthifolius Raddi were examined by normal-phase HPLC. The results revealed higher amounts of tannic acid in the EtOAc-soluble fraction $(79 \%)$ than that in the $n$ $\mathrm{BuOH}$-soluble fraction (45\%).

\section{Acknowledgements}

The author acknowledges Dr. Hideyuki Ito (Division of Pharmaceutical Sciences, Okayama University Graduate School of Medicine, Dentistry and Pharmaceutical Sciences, Tsushima, Okayama 700-8530, Japan) for the NMR measurements.

\section{REFERENCES}

1- W. C. Evans, "Trease and Evans' pharmacognosy", $15^{\text {th }}$ Ed. W.B. Saunders, London, 2002, p. 28.

2- A. M. Editore, "The Macdonald encyclopedia of trees", Macdonald \& Co., London \& Sydney, 1982, p. 166.

3- M. C. R. D. de Carvalho, F. N. T. V. Barca, L. F. Agnez-Lima and S. R. B. de Medeiros, Environ. Mol. Mutag., 42, 185 (2003).

4- M. R. F. de Lima, J. de Souza Luna, A. F. dos Santos, M. C. C. de Andrade, A. E. G. Sant'Ana, J.-P. Genet, B. Marquez, L. Neuville and N. Moreau, J. Ethnopharmacol., $\quad 105,137$ (2006).

5- C. H. Degáspari, N. Waszczynskyj and M. R. M. Prado, Ciênc. Agrotec., Lavras, 29, 617 (2005).
6- S. Johann, M. G. Pizzolatti, C. L. Donnici and M. A. de Resende, Braz. J. Microbiol., 38, 632 (2007).

7- T. Hayashi, K. Nagayama, M. Arisawa, M. Shimizu, S. Suzuki, M. Yoshizaki, N. Morita, J. Nat. Prod., 52, 210 (1989).

8- L. C. Queires, F. Fauvel-Lafetve, S. Terry, A. De la Taille, J. C. Kouyoumdjian, D. K. Chopin, F. Vacherot, L. E. Rodrigues and M. Crepin, Anticancer Res., 26, 379 (2006).

9- M. K. Jain, B.-Z. Yu, J. M. Rogers, A. E. Smith, E. T. A. Boger, R. L. Ostrander and A. L. Rheingold, Phytochemistry, 39, 537 (1995).

10- L. I. F. Jorge and B. E. O. Markmann, Rev. Cienc. Farm. 17, 139 (1996).

11- G. Skopp and G. Schwenker, Z. Naturforsch. B, 41, 1479 (1986).

12- G. Skopp, H. J. Opferkuch and G. Schwenker, Z. Naturforsch. C, 42,7 (1987).

13- E. Stahl, K. Keller and C. Blinn, Planta Med., 48, 5 (1983).

14- F. Teramachi, T. Koyano, T. Kowithayakorn, M. Hayashi, K. Komiyama and M. Ishibashi, J. Nat. Prod., 68, 794 (2005).

15- I. Merfort, Phytochemistry, 31, 2111 (1992).

16- L.-C. Lin, Y.-C. Kuo and C.-J. Chou, J. Nat. Prod., 62, 405 (1999).

17- Y.-H. Chen, F.-R. Chang, M.-C. Lu, P.-W. Hsieh, M.-J. Wu, Y.C. Du and Y.-C. Wu, Molecules, 13, 255 (2008). 
18- T. J. Mabry, K. R. Markham and M. B. Thomas, "The Systematic Identification of Flavonoids", Springer-Verlag, New York (1970).

19- P. K. Agrawal, Phytochemistry, 31, 3307 (1992).

20- T. Fossen, N. A. Frøystein and Ø. M. Andersen, ibid., 49, 1997 (1998).

21- T. Nakanishi, $Y$. Inatomi, $H$. Murata, S. Ishida, Y. Fujino, K. Miura, Y. Yasuno, A. Inada, F. A. Lang and J. Murata, Chem. Pharm. Bull., 55, 334 (2007).
22- A. R. Bilia, J. Mendez and I. Morelli, Pharmaceutica Acta Helvetiae, 71, 191 (1996).

23- S. F. Farag, N. A. El-Emary and M. Niwa, Bull. Pharm. Sci., Assiut University, 21, 1 (1998).

24- P. K. Agrawal, "Carbon-13 NMR of Flavonoids", Elsevier Sciences Publishing Company Inc., New York, 1989, p. 15.

25- A. Dübeler, G. Voltmer, V. Gora, J. Lunderstädt and A. Zeeck, Phytochemistry, 45, 51 (1997). 